\title{
Beobachtungen der Biela-Meteore 1892.
}

Schreiben von Herrn Gymnasial-Oberlehrer F. Plassmann in Warendorf vom 28 . November:

» Nachdem die Tage, für welche die Biela-Meteore zu erwarten waren, abgelaufen sind, wird eine kurze Notiz über das allerdings klägliche Resultat erwünscht sein. Ich hatte hier Nov. $225^{\mathrm{h}} 57^{\mathrm{m}}-7^{\mathrm{h}} 4^{\mathrm{m}}$ kein Meteor bei längerer Be. obachtung von Veränderlichen; nachher bezog sich der Himmel. - Nov. 23, 24, schlechtes Wetter. - Nov. 25 $5^{\mathrm{h}} 3^{2} \mathrm{~m}-6^{\mathrm{h}} 59^{\mathrm{m}}$ und später beim Beobachten von Veränderlichen häufig nach Meteoren gesucht; vielleicht einige schwache gesehen. - Nov. $265^{\mathrm{h}} 48^{\mathrm{m}}-7^{\mathrm{h}} 43^{\mathrm{m}}$ kein Meteor; gegen $18^{\mathrm{h}}$ wieder eine Viertelstunde nachgesehen, aber nichts gefunden. Dagegen theilt mir Herr $\mathcal{F}$. Rheden mit, dass er in Brixen (Tyrol) am 23. Nov. in nicht ganz $3^{\text {h }}$ 48 Meteore eingezeichnet hat, wovon 34 mit ausgeprägter Radiation aus Cassiopeia und Andromeda.* *)

Schreiben von Herrn Prof. F. A. C. Oudemans in Utrecht vom 2. Dec.:

*Wir hatten in den letzten Wochen hier fast anhaltend

Nebel. Am 27. November war es eine kleine halbe Stunde heiter (etwa von $\mathrm{II}^{\mathrm{h}} 30^{\mathrm{m}}$ bis $\mathrm{I}^{\mathrm{h}} 5^{0^{\mathrm{m}}}$ M. Z. Utrecht), in welchem Zeitraum ich jedoch keine einzige Sternschnuppe gesehen habe. *

*) Auch in New York und Cambridge U.S. ist am 23. Nov. Abends ein reicher Sternschnuppenfall, mehr als 200 in 40 Minuten, aus Radiant $10^{\circ}$ nordöstlich vom Comet Holmes, gesehen worden. Vgl. Astr. Journ. 280.

Kr.

\section{Berichtigungen von Druckfehlern in den Abzählungen der Bonner Durchmusterungssterne.}

$\mathrm{Zu}$ meiner Freude werden in neuerer Zeit meine $\mathrm{Ab}$. zählungen der Bonner Durchmusterungen vielfach benutzt. Es scheint mir deshalb nicht unnöthig, zu bemerken, dass in den abgekürzten Tabellen, welche in den Sitzungsberichten der Münchener Akademie erschienen sind, einige Druckfehler stehen geblieben sind. In den ausführlichen Tabellen im II. Bande der neuen Annalen der Münchener Sternwarte sind bisher noch keine gefunden worden und es ist deshalb rathsam, bei etwaigen Zweifeln diese letzteren zu Rathe zu ziehen.

Die bisher bemerkten Druckfehler beziehen sich auf die nördliche Durchmusterung und sind folgende, wenn von den in der auf die südliche Durchmusterung bezüglichen Arbeit publicirten Druckfehlern abgesehen wird:

S. 533, Z. 7 v. u. Verticalreihe I muss stehen

-533, 17 v.u.

$533,>6$ v. o.

, 533, 8 v. 0 .

-535, 17 v.u.

-537, 8 v.u.

-537, r v.u. verstorbenen Kleiber bemerkt worden. Hiermit erledigen sich die von Herrn Ristenpart in seinen kürzlich erschienenen »Untersuchungen über die Constante der Praecession etc. gemachten Bemerkungen in allerdings anderer Weise als dort angegeben worden ist.

Ich habe s. $Z$. ausdrücklich angegeben (S. 525), dass sich bei der Abzählung der nördlichen Durchmusterung ein wohl für alle Fälle völlig belangloses Deficit von 1 I Objecten ergeben hat. Es wäre in gar keinem Verhältnisse zu dem zu erlangenden Erfolg gewesen, dieses Deficit wegschaffen zu wollen. Dasselbe ist dadurch entstanden, dass einige Zahlen in dem benutzten Exemplare mangelhaft waren und demzufolge auch bei doppeltem Nachzählen übersehen werden konnten. Herr Ristenpart hat nun a. a. O. eines der I I fehlenden Objecte gefunden. In $\delta+8^{\circ}$, Rubrik $2^{\mathrm{h}} 40^{\mathrm{m}}-$ $3^{\text {h }} 20^{\mathrm{m}}$ sind 2, nicht I Stern von der Grösse 1.0 bis 6.5. Indem ich diese Correctur dankbar acceptire, glaube ich aber nicht, dass es angemessen ist, alle gebildeten Summen um eine Einheit zu corrigiren, bevor nicht auf irgend einem Wege das ganze genannte Deficit fortgeschafft ist. Bei dem höchst gleichgültigen Charakter desselben wird dies wohl nur durch eine Häufung von glücklichen Zufällen zu erwarten sein.

Diese Druckfehler sind theils von mir, theils von dem

München 1892 Nov. 15.

H. Seeliger.

Comet 1893...(Brooks 1892 Nov. 19). Nach Astr. Journ. 280 ist der Comet von Brooks bereits am 19. Nov. $\left(\alpha=12^{\mathrm{h}} 5^{6^{\mathrm{m}}}, \delta=+12^{\circ} 59^{\prime}\right)$ entdeckt worden. Die Angabe im Entdeckungstelegramm, A. N. 3130 p. 167 , ist hiernach zu berichtigen; ferner ist in dem letzteren die hier schon gleich anfangs bezweifelte Angabe über das Aussehen des Cometen zu lesen : $\gg$ Circular, diameter $=$ I’, ro mag., tolerably well defined nucleus, no tail . $K r$.

\section{Inhalt :}

Zu Nr. 3134-35. G. Lorentzen. Ueber die Untersuchung der Scalen eines Heliometers. 2I7. - F. Holetschek. Ueber die Beobachtung und die Berechnung von Cometen-Helligkeiten. 239. - Beobachtungen des Cometen 1893...(Brooks 1892 Nov. 19). 245. - Beobachtungen der Biela-Meteore 1892. 247. - H. Seeliger. Berichtigungen von Druckfehlern in den Abzählungen der Bonner Durchmusterungssterne. 247. - Comet 1893 ...(Brooks 1892 Nov. 19). 247. 